ARTIGO ORIGINAL ORIGINAL ARTICLE

\title{
Custo-efetividade de cateteres periféricos com plataforma de estabilização integrada sob a perspectiva hospitalar no Brasil
}

\author{
Cost-effectiveness of peripheral catheters with integrated \\ stabilization platform under the perspective of hospitals in Brazil
}

Luciana Lopes Mensor', Daniele Giorgio de Aguiar², Camila Pepe Ribeiro de Souza ${ }^{3}$

DOI: 10.21115/JBES.v8.n1.p16-23

\section{Palavras-chave:}

custo-efetividade, cateteres venosos periféricos, plataforma de estabilização, flebite, deslocamento.

\section{Keywords:}

cost-effectiveness, peripheral catheters, stabilization platform, phlebitis, dislodgement.

\begin{abstract}
RESUMO
A presente avaliação tem como finalidade determinar a custo-efetividade de cateteres periféricos com dispositivo de segurança, válvula antirrefluxo e plataforma de estabilização integrada durante seu uso em pacientes hospitalizados e submetidos à terapia de infusão, sob a perspectiva da Saúde Suplementar do Brasil. Foi elaborado modelo analítico de decisão para estimar a razão de custo-efetividade incremental (RCEI), em horizonte de 96 horas, entre cateteres periféricos com plataforma de estabilização integrada versus dispositivos sem plataforma de estabilização. Os desfechos clínicos aplicados ao modelo foram taxa de sucesso da punção venosa e taxa de permanência do cateter, obtidos através de revisão sistemática da literatura. A estimativa de custos médicos diretos totais para o tratamento das complicações associadas à punção venosa foi elaborada por opinião de especialistas, e coletada a partir de de bases de dados secundárias. O resultado para o Caso Base do modelo evidenciou economia de recursos financeiros de $\mathrm{R} \$ 138,31$ por complicações evitadas e $\mathrm{R} \$ 558,33$ por punção venosa com sucesso ou novas punções evitadas. O estudo indica que o uso de cateteres periféricos com plataforma de estabilização integrada pode constituir uma alternativa viável aos hospitais brasileiros, gerando redução de custos totais de tratamento e ganhos em efetividade clínica, especialmente em função da menor tendência de surgimento de eventos adversos decorrentes da mobilização inadvertida dos cateteres periféricos ao longo do tempo.
\end{abstract}

\begin{abstract}
The current evaluation aims at determining the cost-effectiveness of peripheral catheters with securement device, blood control septum and integrated stabilization platform in use in patients admitted to hospitals and submitted to infusion therapy, under the perspective of Supplementary HealthCare in Brazil. A decision tree was elaborated for estimating the incremental cost-effectiveness ratio (ICER) between two types of peripheral catheters, with or without an integrated stabilization platform, in a time horizon of 96 hours. Clinical outcomes applied to the model were obtained from systematic review of literature published up to 31.08.2015 and include rates of venipuncture success and catheter indwell time. The average of total direct medical costs for treatment of adverse events was estimated by expert opinion and data was collected from secondary databases. The Base Case results reflect cost-savings of about 138,31 Brazilian Reais per avoided complications and 558,33 Brazilian Reais per successful venipuncture or new venipunctures avoided. Ours study points out the use of peripheral catheters with stabilization platforms integrated to the device constitutes feasible alternatives for Brazilian private hospitals. Their use can generate reduction of total treatment costs and gains in effectiveness specially due to lower tendencies for development of securement-related adverse reactions, caused by inadvertent mobilization of peripheral access over time.
\end{abstract}

Recebido em: 04/02/2016. Aprovado para publicação em: 21/03/2016

1 Gerente de Farmacoeconomia, Laboratórios B. Braun S/A, Rio de Janeiro, Brasil.

2 Daniele Giorgio de Aguiar, Gerente de Produto, Laboratórios B. Braun S/A, Rio de Janeiro, Brasil.

3 Diretora de Projetos, Sense Company, São Paulo, Brasil.

Instituição onde o trabalho foi executado: Sense Company, São Paulo, Brasil, Laboratórios B.Braun S.A., Rio de Janeiro, Brasil Informação sobre auxilio recebido sob a forma de financiamento, equipamentos ou medicamentos: Esta Avaliação de Tecnologias em Saúde foi integralmente financiada pelos Laboratórios B.Braun S.A. Os autores Luciana Lopes Mensor e Daniele Giorgio de Aguiar trabalham nos Laboratórios B.Braun S.A. Camila Pepe Ribeiro de Souza trabalha na Sense Company empresa contratada pelos Laboratórios B.Braun S.A para elaboração dos fundamentos deste artigo.

Congressos onde o estudo foi apresentado: o estudo foi submetido e aceito para apresentação no $21^{\circ}$. Congresso Anual da Sociedade Internacional de Farmacoeconomia (ISPOR), que ocorrerá em maio de 2016, Washington, DC, e no $4^{\circ}$. Congresso Mundial em Acesso Vascular (WoCoVa), que ocorrerá em junho de 2016, Lisboa, Portugal.

Autor correspondente: Luciana Lopes Mensor, Av. Eugênio Borges 1092, São Gonçalo, RJ, CEP 24751-000. E-mail: luciana.mensor@ bbraun.com; lucianamensor@gmail.com 


\section{Introdução}

A punção venosa com uso de cateteres periféricos ocorre em, pelo menos, um terço dos pacientes admitidos em hospitais (Jacinto et al., 2014). Por tratar-se de procedimento invasivo e de uso frequente, a mesma pode suscitar eventos adversos e complicações locais ou sistêmicas nos pacientes (Jacinto et al., 2014; Souza et al., 2015). A flebite mecânica é a complicação local mais comum, sendo decorrente de

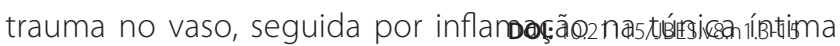
venosa, que pode culminar em trombose e sepse (Campos, 2015; Magerote et al., 2011). Outras complicações comuns incluem deslocamento do cateter, infiltração e extravasamento (Schears, 2006).

Os fatores desencadeadores destas reações adversas são vários, tais como tipo de material, calibre, comprimento e espessura do cateter, número de inserções em um mesmo paciente, local de inserção e período de manutenção do cateter (Pasalioglu \& Kaya, 2014). O tipo do cateter empregado, uso de plataformas estabilizadoras e de adesivos transparentes podem, no entanto, reduzir a movimentação do cateter e, consequentemente, diminuir as taxas de complicações associadas a seu uso (Bausone-Gazda et al., 2010).

No Brasil, estudos realizados com pacientes hospitalizados reportam taxas de incidência de flebite mecânica entre 3\% e 20,6\% (Magerote et al., 2011). Porém, a Infusion Nurses Society (INS) determina como 5\% a taxa máxima aceita para a ocorrência de flebite, estabelecendo padrões que recomendam que o cateter seja estabilizado no seu ponto de inserção para preservar o acesso venoso (Infusion Nurses Society, 2006; Jacinto et al., 2014; Magerote et al., 2011). Também recomenda que os produtos utilizados para tal estabilização devem ter sua eficiência comprovada, bem como sua durabilidade, facilidade de uso e custo viável. Tais recomendações indicam que os dispositivos de estabilização integrados ao cateter compõem o método preferencial, devendo ser utilizados sempre que possível (Bausone-Gazda et al., 2010; Infusion Nurses Society, 2006).

Assim, o objetivo da avaliação apresentada neste artigo foi determinar a custo-efetividade de cateteres venosos periféricos com dispositivo de segurança e plataforma de estabilização integrada versus cateteres periféricos com dispositivo de segurança, porém sem plataforma de estabilização. A perspectiva hospitalar brasileira foi a escolhida para a análise.

\section{Métodos}

O produto em avaliação trata-se de um cateter venoso periférico constituído de poliuretano, com mecanismo de segurança passivo, além de válvula antirrefluxo e plataforma de estabilização integrada, em sistema fechado. A plataforma de estabilização integrada visa minimizar a movimentação do cateter e auxiliar na redução de irritações na parede do vaso. Ainda, a conexão em Luer minimiza a contaminação do dispositivo por contato (Laboratórios B.Braun S/A, 2013).

Foi desenvolvida uma análise de custo efetividade através de modelo analítico de decisão, elaborado sob a perspectiva da Saúde Suplementar do Brasil (hospitais privados), comparando o uso de cateteres periféricos de segurança com e sem plataforma de estabilização integrada. Como horizonte temporal, foi selecionado um cenário de curto prazo de até 96 horas após punção venosa periférica.

Os desfechos clínicos e dados epidemiológicos utilizados no modelo econômico foram coletados por meio de revisão sistemática da literatura científica. As buscas eletrônicas foram realizadas até o final de agosto de 2015. Estas buscas seguiram a hierarquia de prioridade sugerida pela Diretriz para Elaboração de Pareceres Técnico-Científicos do Ministério da Saúde (BRASIL, 2011) e foram realizadas em todas as bases obrigatórias (Registro Cochrane Central de Ensaios Controlados, The Cochrane Library, MEDLINE via Pubmed, Literatura Latino-Americana e do Caribe em Ciências da Saúde - LILACS e Centre for Reviews and Dissemination - (RD), conforme a pergunta estruturada no formato PICO, descrita no Quadro 1.

Foi ainda realizada busca complementar por estudos econômicos nas bases de dados Pubmed/MEDLINE, CRD, Biblioteca Virtual em Saúde - Economia da Saúde (BVS-ECOS), e em sítios eletrônicos de Agências de Avaliação de Tecnologias em Saúde, instituições correlatas e suas bases de dados.

$\mathrm{Na}$ construção de estratégias de buscas, descritores, palavras-chave e termos MeSH foram utilizados para cada base de dado especificamente. Não foram utilizados limites temporais, tampouco filtros para revisões sistemáticas ou ensaios clínicos para que as buscas fossem mais abrangentes. Apenas artigos nos idiomas português, inglês, francês, espanhol ou italiano foram incluídos.

Os estudos incluídos na revisão foram avaliados conforme a Classificação de Nível de Evidência da Oxford Centre for Evidence Based Medicine (OXFORD, 2009). Para avaliação da qualidade metodológica das revisões sistemáticas incluídas, foi utilizada a ferramenta AMSTAR (Shea et al., 2007, 2009),

Quadro 1. Pergunta estruturada no formato PICO da revisão sistemática.

\begin{tabular}{ll}
\hline P - População & $\begin{array}{l}\text { Pacientes hospitalizados com acesso } \\
\text { venoso por meio de cateter periférico. }\end{array}$ \\
\hline I- Intervenção & $\begin{array}{l}\text { Cateteres periféricos com } \\
\text { plataforma de estabilização. }\end{array}$ \\
\hline C - Comparação & $\begin{array}{l}\text { Cateteres periféricos sem } \\
\text { plataforma de estabilização. }\end{array}$ \\
\hline O- Desfechos & Desenvolvimento de flebite mecânica. \\
\hline $\begin{array}{l}\text { Tipos de estudos } \\
\text { incluídos }\end{array}$ & $\begin{array}{l}\text { Metanálises, revisões sistemáticas, ensaios } \\
\text { Clínicos e estudos observacionais. }\end{array}$ \\
\hline
\end{tabular}


e para os ensaios clínicos randomizados controlados foram avaliados conforme a metodologia proposta por Jadad e colaboradores (Jadad et al., 1996).

O modelo econômico foi desenvolvido utilizando-se o software TreeAge Pro 2013 (TreeAge Software Inc., Williamstown, Estados Unidos), a fim de estimar a razão de custo-efetividade incremental (RCEI) entre as duas tecnologias empregadas em hospitais no Brasil: a) cateter venoso periférico com dispositivo de segurança, válvula antirrefluxo e plataforma de estabilização integrada; b) cateteres venosos periféricos com dispositivo de segurança, com ou sem válvula antirrefluxo e sem plataforma de estabilização.

O modelo elaborado simula um coorte hipotético de pacientes internados em hospitais da rede privada do Brasil, sob regime de terapia infusional por acesso periférico, e submetidos ao uso dos comparadores (Figura 1). O racional para o modelo foi que, após a inserção do cateter em acesso periférico, existe um risco de desenvolvimento de reações adversas de segurança (no inglês, "securement-related adverse reactions"), que é aumentado quando os dispositivos empregados possuem pouca estabilização, gerando eventos que incluem deslocamentos, infiltração, oclusão, flebite mecânica, entre outros. Tais reações contribuem para redução da taxa de permanência do cateter para tempos menores do que as 72 a 96 horas preconizadas pela maior parte dos hospitais brasileiros.

Já que o modelo contempla horizonte temporal inferior a 1 (um) ano, não foi considerada taxa de desconto (BRASIL, 2009). Ainda, foram considerados como comparadores apenas cateteres periféricos com dispositivos de segurança, haja vista que a Norma Regulamentadora 32/2005, publicada pelo Ministério do Trabalho e Emprego, estabelece a obrigatoriedade deste tipo de mecanismo para conferir proteção aos trabalhadores da saúde no caso de acidentes com materiais perfuro cortantes potencialmente contaminados com material biológico infectado (MTE, 2005).

Adicionalmente, a análise comparativa evidenciada refere-se exclusivamente à presença ou não de plataforma de estabilização integrada nos cateteres periféricos. Por conseguinte, a presença ou ausência de válvula antirrefluxo no cateter não foi considerada como característica relevante para os desfechos selecionados, e os cateteres que possuem ou não tal dispositivo foram agrupados em uma única categoria. Assim, para fins desta avaliação, serão denominados "Dispositivos de primeira geração" os cateteres periféricos com dispositivo de segurança e sem válvula antirrefluxo nem plataforma de estabilização. Como "Dispositivos de segunda geração", aqueles cateteres com dispositivo de segurança e válvula antirrefluxo, mas sem plataforma de estabilização. E como "Dispositivos de terceira geração", foi considerado apenas um cateter periférico que contém dispositivo de segurança, válvula antirrefluxo e plataforma de estabilização integrada (tecnologia em avaliação, Introcan Safety ${ }^{\circledR}$, Laboratórios B.Braun S.A.)

Os custos estimados aplicados ao modelo foram baseados em preços coletados de bases secundárias, considerando valores constantes das listas de Preços Fábrica (PF), disponíveis para uma taxa de Imposto sobre Circulação de Mercadorias e Serviços (ICMS) de 18\%. Custos indiretos e custos não médicos não foram considerados por não se

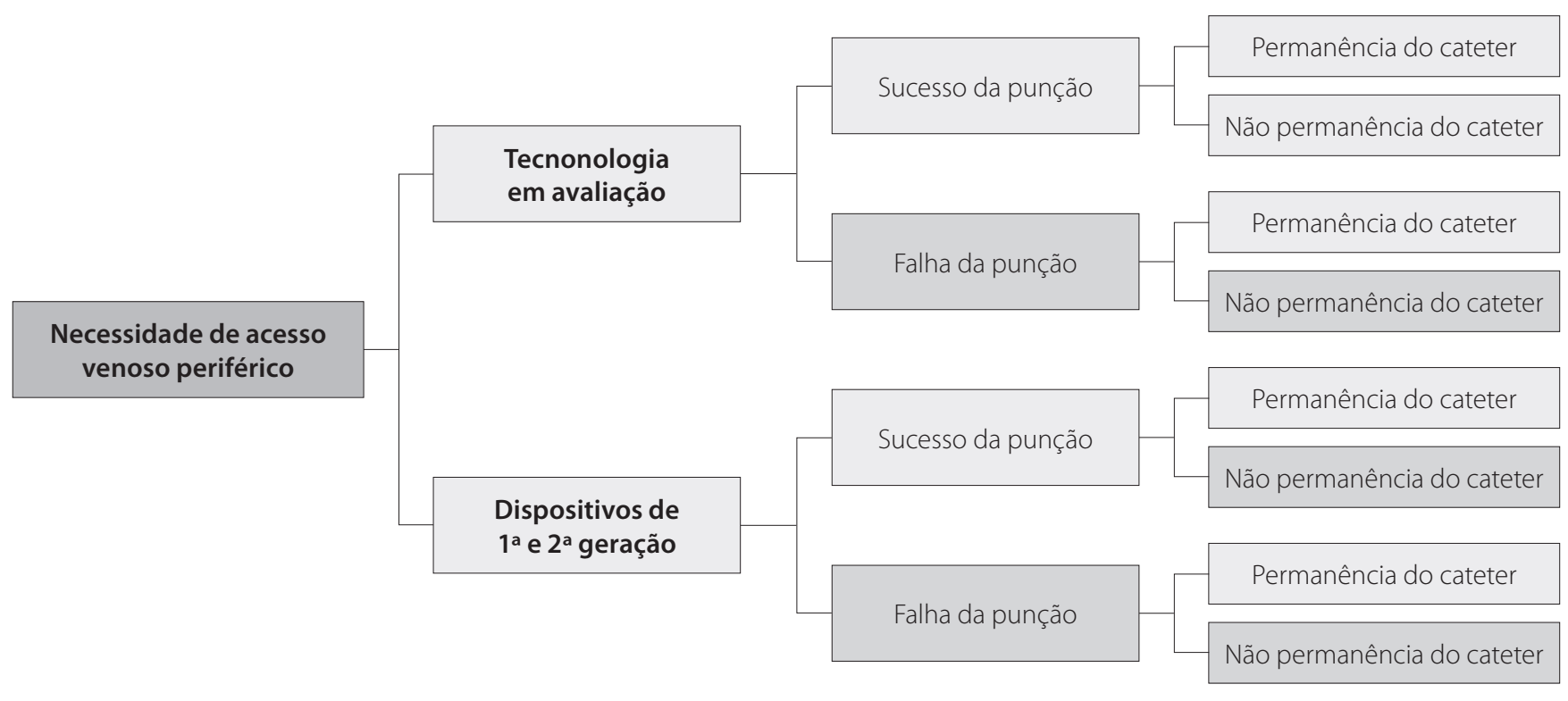

Figura 1. Modelo analítico de decisão elaborado para estimar a RCEl entre as duas tecnologias para cateterização intravenosa periférica em avaliação. 
adequarem à perspectiva selecionada. Os recursos de saúde considerados se referem a procedimentos e exames relacionados ao tratamento dos eventos adversos à punção venosa com cateteres periféricos, ponderados a partir das respectivas taxas de ocorrência destas reações, conforme o comparador empregado.

Os exames foram custeados conforme a Classificação Brasileira Hierarquizada de Procedimentos Médicos da Associação Médica Brasileira (CBHPM, 2014). Os preços de aquisição dos dispositivos médicos e produtos para a saúde foram baseados na Revista Eletrônica SIMPRO, para data base de junho de 2015 (SIMPRO, 2015) ou da Revista Brasíndice, para a mesma data base de 2015 (BRASínDICE, 2015). Os preços de medicamentos foram coletados a partir da Lista da Câmara de Regulação de Medicamentos, também com data base de junho de 2015 (CMED, 2015).

Os resultados comparativos das estratégias de tratamento foram avaliados pela RCEI, definida para duas alternativas de tratamentos específicos como o custo adicional proporcionado pela alternativa em análise dividido pelo ganho adicional em saúde alcançado pela mesma. Foi considerado como limiar de custo-efetividade o valor do PIB per capita no Brasil em 2014, ou R\$27.229,00 (IBGE, 2014). Para resultados de RCEI negativa, foi considerado que a alternativa proposta atingiria os objetivos com economia de recursos. Como forma de mensurar as incertezas relativas aos resultados, foram conduzidas análises de sensibilidade de preços para cada cenário proposto.

Como "Caso Base", ou Cenário 1, foi definida a comparação entre o Dispositivo de 3a. geração, ou Tecnologia em Avaliação, e os dispositivos de $1^{\mathrm{a}}$ e $2^{\mathrm{a}}$ geração. A análise desenvolvida neste cenário foi a análise de custo-efetividade (ACE), já que os dados de eficácia empregados no modelo diferem entre os comparadores. Assim, foram consideradas as taxas de sucesso da primeira punção e as taxas de ocorrência de complicações diferentes entre os comparadores, além dos preços dos cateteres conforme preços de lista registrados no Brasil.
Como cenário alternativo, ou cenário 2, os preços dos cateteres foram zerados no modelo, como tentativa de contornar as taxas de desconto aplicadas como preços de mercado.

\section{Resultado}

A revisão sistemática de literatura foi realizada com dois revisores e localizou, após a realização da busca nas bases de dados, 371 títulos (incluindo duplicatas). Aplicados os critérios de elegibilidade, os revisores selecionaram 7 estudos para leitura na íntegra. Desses, 4 estudos foram selecionados e incluídos nesta revisão. Em relação às buscas econômicas, não foram selecionados estudos para a leitura na íntegra.

Dados de eficácia relacionados à efetividade dos comparadores foram obtidos de dois estudos, sendo o estudo randomizado de Bausone-Gazda e colaboradores publicado em 2010 (Bausone-Gazda et al., 2010) e o estudo observacional de Schears de 2006 (Schears, 2006). Os desfechos clínicos "taxa de sucesso da punção venosa" e "taxa de permanência do cateter em até 96 horas", estão demonstrados na Tabela 1. A metanálise publicada em 2015 pelo grupo de Marsh (Marsh et al., 2015), principal evidência localizada na etapa de revisão sistemática, não foi aplicada diretamente ao modelo pois, no que se refere aos parâmetros elegidos, a mesma também referencia o estudo de Bausone-Gazda.

Os valores unitários dos comparadores incluídos na avaliação são descritos na Tabela 2 e englobam médias aritméticas entre diferentes produtos comercializados no país, para uma mesma categoria ou geração de dispositivos. O padrão de tratamento para as complicações associadas foi elaborado a partir de opinião de especialistas e os custos totais de cada tratamento podem ser visualizados nas Tabelas 3 e 4 . Após a aplicação de todos os vetores supracitados de custo e eficácia clínica dentro do modelo econômico desenvolvido, foram atingidos os resultados das RCEl para cada cenário considerado (Tabelas 5 e 6).

As RCEl observadas no Caso Base traduzem o fato de que, para cada punção com sucesso, são economizados o equiva-

Tabela 1. Desfechos clínicos aplicados ao modelo analítico de decisão.

\begin{tabular}{lccc}
\hline Desfecho clínico & $\begin{array}{c}\text { Dispositivos com plataforma } \\
\text { de estabilização integrada }\end{array}$ & $\begin{array}{c}\text { Dispositivos de 1a e 2 }{ }^{\text {a }} \text { geração } \\
\text { (sem plataforma de estabilização) }\end{array}$ & Referência \\
\hline $\begin{array}{l}\text { Taxa de sucesso da primeira } \\
\text { punção venosa }(t=0)(p=0,036)\end{array}$ & $90,7 \%$ & $82,2 \%$ & Bausone-Gazda et al., 2010 \\
\hline $\begin{array}{l}\text { Taxa de deslocamento do } \\
\text { cateter ( } t=96 \text { horas) }(p=0,017)\end{array}$ & $2 \%$ & $12 \%$ & Bausone-Gazda et al., 2010 \\
\hline $\begin{array}{l}\text { Taxa de flebite mecânica } \\
(t=96 \text { horas) ( } p<0,001)\end{array}$ & $0,7 \%$ & $3,6 \%$ & Schears, 2006 \\
\hline $\begin{array}{l}\text { Taxa total de complicações* } \\
(t=96 \text { horas) }(p<0,001)\end{array}$ & $16 \%$ & $47,6 \%$ & Schears, 2006 \\
\hline
\end{tabular}

* Inclui todas complicações elencadas no estudo original (Schears, 2006), sendo elas: infiltração, extravasamento, oclusão, deslocamento e flebite mecânica. 
Tabela 2. Valores unitários considerados para dispositivos de 1a, 2a e $3^{\mathrm{a}}$ gerações.

\begin{tabular}{|c|c|c|}
\hline Dispositivos & Preço fábrica unitário & Fonte \\
\hline Dispositivos de $1^{\mathrm{a}}$ geração & $\mathrm{R} \$ 57,29 *$ & BRASÍNDICE, Nr. 835 \\
\hline Dispositivos de $2^{\mathrm{a}}$ geração & $R \$ 57,65^{*}$ & BRASÍNDICE, Nr. 835 \\
\hline Dispositivo de $3^{a}$ geração (Tecnologia em avaliação) & $\mathrm{R} \$ 69,72^{* *}$ & BRASÍNDICE, Nr. 835 \\
\hline
\end{tabular}

Tabela 3. Custos totais associados a troca de acesso venoso.

\begin{tabular}{|c|c|c|c|c|c|}
\hline & $\%$ uso & Quantidade & Custo unitário* & Custo total & Fonte \\
\hline Honorários de enfermagem & $100 \%$ & 1 & $\mathrm{R} \$ 13,22$ & $R \$ 13,22$ & COFEN \\
\hline Luva procedimento & $100 \%$ & 2 & $\mathrm{R} \$ 0,30$ & $R \$ 0,60$ & SIMPRO \\
\hline Máscara descartável & $100 \%$ & 1 & $\mathrm{R} \$ 0,23$ & $\mathrm{R} \$ 0,23$ & SIMPRO \\
\hline Compressa gaze estéril & $100 \%$ & 1 & $R \$ 0,53$ & $R \$ 0,53$ & SIMPRO \\
\hline Fita hipoalergênica micropore & $50 \%$ & 1 & $R \$ 36,83$ & $\mathrm{R} \$ 18,42$ & SIMPRO \\
\hline Curativo de filme transparente & $50 \%$ & 1 & $\mathrm{R} \$ 5,87$ & $\mathrm{R} \$ 2,93$ & SIMPRO \\
\hline Álcool 70 & $100 \%$ & 1 & $R \$ 2,37$ & $R \$ 2,37$ & SIMPRO \\
\hline Seringa de $5 \mathrm{ml}$ & $100 \%$ & 1 & $R \$ 1,55$ & $\mathrm{R} \$ 1,55$ & BRASÍNDICE \\
\hline Agulha 40x12 c/ disp. segurança & $100 \%$ & 1 & $R \$ 6,02$ & $\mathrm{R} \$ 6,02$ & BRASÍNDICE \\
\hline Cloreto de sódio 0,9\% 10 ml & $100 \%$ & 1 & $\mathrm{R} \$ 0,58$ & $\mathrm{R} \$ 0,58$ & CMED \\
\hline Polifix & $100 \%$ & 1 & $R \$ 30,25$ & $R \$ 30,25$ & BRASÍNDICE \\
\hline CUSTO TOTAL - Punção & & & & $R \$ 76,70$ & \\
\hline
\end{tabular}

*Os valores praticados foram obtidos da lista de preços da Câmara de Regulamentação do Mercado de Medicamentos (CMED), COFEN (COFEN, 2012) e revista SIMPRO Hospitalar (SIMPRO, 2015).

Tabela 4. Custo do manejo de complicações totais (infiltração, extravasamento, oclusão, deslocamento, flebite mecânica).

\begin{tabular}{lccccc}
\hline & $\%$ uso & Quantidade & Custo unitário* & Custo total & Fonte \\
\hline Honorários de enfermagem & $100 \%$ & 1 & $R \$ 13,22$ & $R \$ 13,22$ & COFEN \\
\hline Agulha 40x12 com dispositivo de segurança & $100 \%$ & 1 & $R \$ 6,02$ & $R \$ 6,02$ & BRASINDICE \\
\hline Seringa de $5 \mathrm{ml}$ & $100 \%$ & 1 & $\mathrm{R} \$ 1,55$ & $\mathrm{R} \$ 1,55$ & $\mathrm{BRASINDICE}$ \\
\hline Cloreto de sódio 0,9\% $10 \mathrm{ml}$ & $100 \%$ & 1 & $\mathrm{R} \$ 0,58$ & $\mathrm{R} \$ 0,58$ & $\mathrm{BRASINDICE}$ \\
\hline Troca do acesso venoso periférico & $100 \%$ & 1 & $\mathrm{R} \$ 76,70$ & $\mathrm{R} \$ 76,70$ & $\mathrm{Calculado}$ \\
\hline CUSTO TOTAL - Flebite mecânica & & & & $\mathrm{R} \$ 98,07$ & \\
\hline
\end{tabular}

*Os valores praticados foram obtidos da lista de preços da Câmara de Regulamentação do Mercado de Medicamentos (CMED), COFEN (COFEN, 2012) e revista SIMPRO Hospitalar (SIMPRO)

lente a $\mathrm{R} \$ 558,33$ ou $\mathrm{R} \$ 138,31$ para cada complicação evitada que possa reduzir a taxa de permanência total do cateter periférico, respectivamente.

Na análise de cenários, desconsiderando-se no modelo o efeito de preços de aquisição dos cateteres, observa-se que a economia de recursos financeiros ainda se mantém expressiva (R\$ 117,06 por complicação evitada e $R \$ 472,55$ por sucesso de punção venosa). Tal economia decorre direta e inequivocamente à menor taxa de eventos adversos associada aos cateteres com plataforma integrada, que levam consequentemente a um menor dispêndio financeiro relacionado ao tratamento de tais eventos. Ou seja, esta análise é importante pois preços de aquisição são extremamente variáveis e sensíveis a situações mercadológicas.

Tendo seu efeito direto desconsiderado, chega-se a valores de economia referentes exclusivamente às menores taxas de eventos adversos originárias de uma eventual incorporação da inovação em saúde.

Tais dados combinados denotam uma relação de custo-efetividade extremamente favorável ao uso de dispositivos 
Tabela 5. Análise de custo-efetividade para o caso base, comparando-se dispositivos com e sem plataforma de estabilização com relação às taxas de sucesso da punção e de complicações gerais.

\begin{tabular}{llcc}
\hline & & \multicolumn{1}{c}{ Efetividade total } \\
\cline { 2 - 4 } & Custo total & $\begin{array}{c}\text { Taxa de sucesso da } \\
\text { punção (t = 0) }\end{array}$ & $\begin{array}{c}\text { Taxa de permanência } \\
\text { do cateter (t - 96h) * }\end{array}$ \\
\hline $\begin{array}{l}\text { Dispositivos com mecanismo de segurança e } \\
\text { plataforma de estabilização integrada } \\
\text { (tecnologia em avaliação) }\end{array}$ & $R \$ 199,99$ & $90,70 \%$ & $76,19 \%$ \\
\hline Dispositivos de 1a e 2a geração (sem plataforma de estabilização) & $R \$ 245,55$ & $82,54 \%$ & $43,25 \%$ \\
\hline Incremental & $-R \$ 45,56$ & $8,16 \%$ & $32,94 \%$ \\
\hline RCEl & & $-R \$ 558,33$ & $-R \$ 138,31$ \\
\hline
\end{tabular}

RCEl: Razão de custo-efetividade incremental. * inverso da taxa de complicações gerais.

Tabela 6. Análise de custo-efetividade para o cenário alternativo, em que os preços dos cateteres foram zerados, mantendo-se os desfechos clínicos respectivos como no caso base.

\begin{tabular}{|c|c|c|c|}
\hline & \multirow[b]{2}{*}{ Custo total } & \multicolumn{2}{|c|}{ Efetividade total } \\
\hline & & $\begin{array}{l}\text { Taxa de sucesso da } \\
\text { punção }(t=0)\end{array}$ & $\begin{array}{l}\text { Taxa de permanência } \\
\text { do cateter }(t=96 h) *\end{array}$ \\
\hline $\begin{array}{l}\text { Dispositivos com mecanismo de segurança e } \\
\text { plataforma de estabilização integrada } \\
\text { (tecnologia em avaliação) }\end{array}$ & $\mathrm{R} \$ 107,19$ & $90,70 \%$ & $76,19 \%$ \\
\hline Dispositivos de $1^{\mathrm{a}}$ e $2^{\mathrm{a}}$ geração (sem plataforma de estabilização) & $\mathrm{R} \$ 145,75$ & $82,54 \%$ & $43,25 \%$ \\
\hline Incremental & $-R \$ 38,56$ & $8,16 \%$ & $32,94 \%$ \\
\hline RCEI & & $-R \$ 472,55$ & $-\mathrm{R} \$ 117,06$ \\
\hline
\end{tabular}

RCEl: Razão de custo-efetividade incremental. * inverso da taxa de complicações gerais.

com plataforma de estabilização integrada, já que os custos totais de tratamento com este comparador, obtidos pelo modelo para um horizonte de 96 horas, foram significativamente menores do que os obtidos para o grupo sem plataforma. O norteador de custos majoritário para este resultado foi exatamente a menor incidência de eventos adversos associados à punção venosa quando empregada plataforma de estabilização integrada ao cateter, que se traduz em menor necessidade de tratamento de tais reações.

As análises de sensibilidade realizadas demonstram que, no caso base, considerando os preços fábrica dos produtos em comparação, para igualar o custo incremental a zero, o cateter em avaliação poderia custar até $\mathrm{R} \$ 103,94$. Ao excluir-se o efeito de preço de aquisição do modelo, para igualar a RCEl, o produto em questão poderia custar até $R \$ 28,97$ a mais do que o seu comparador tradicional, sem plataforma de estabilização integrada (Figuras 2 e 3).

\section{Discussão}

A taxa de falha referente a cateterização periférica é significativa, tendo sido relatada na literatura científica como variando entre 33 e 69\%. Existem diversos fatores relacionados à terapia IV ou às características do paciente ou mesmo do próprio cateter que dificultam a obtenção do acesso venoso periférico e influenciam na ocorrência de complicações. Remoção acidental do dispositivo, flebites, oclusão, infiltração, infecção são algumas das muitas razões pelas quais a cateterização periférica pode não ser bem sucedida. A incidência de flebites em pacientes hospitalizados reportada na literatura mostra-se variável entre 3\% e 20,6\% (Magerote et al., 2011; Marsh et al., 2015).

No entanto, estudos internacionais publicados recentemente chamam atenção ao fato de que dispositivos complataformas de estabilização já integradas, ou seja, com pontos de fixação, ancoragem ou clipes que façam parte da sua estrutura, com intuito de melhor estabilizar os cateteres periféricos em seu local de inserção, podem aumentar a capacidade de fixação dos mesmos, minimizando assim suas movimentações não intencionais e reduzindo as complicações decorrentes destas. A INS, por exemplo, já recomenda que dispositivos dessa natureza, prontos para o uso, sejam utilizados sempre que possível, ao invés de adesivos para fixação (Marsh et al., 2015).

Através de revisão sistemática da literatura conduzida para fomentar o modelo com dados de eficácia, foi avaliado se o uso de cateteres periféricos com plataforma de estabilização integrada preveniria a ocorrência, em última análise, de flebite 


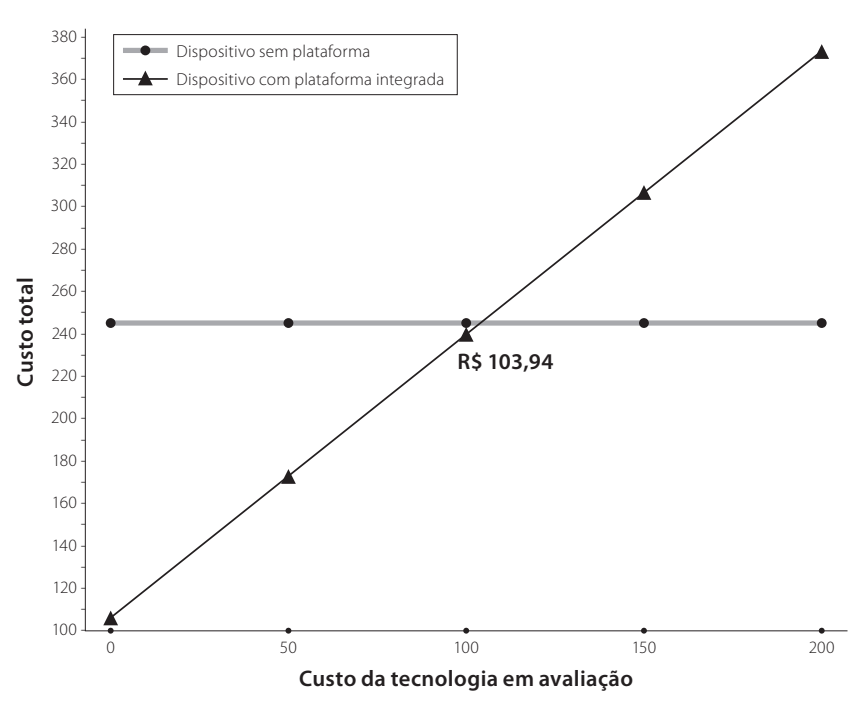

Figura 2. Análise de sensibilidade de preços para o caso base.

mecânica em pacientes hospitalizados com acesso venoso, quando comparado a dispositivos sem nenhum mecanismo que confira fixação, além de adesivos externos. Ao final do processo de revisão, foram incluídos quatro estudos que avaliaram cateteres periféricos com abas de fixação, integradas ou não ao cateter, entre estes uma revisão sistemática (Marsh et al., 2015), dois ensaios clínicos randomizados (Bausone-Gazda et al., 2010; Tamura et al., 2014) e um estudo de coorte (Schears, 2006). Embora os estudos randomizados incluídos (Bausone-Gazda et al., 2010; Tamura et al., 2014) não tenham evidenciado diferença significativa relacionada a taxa de incidência de deslocamento do cateter, flebite mecânica ou extravasamento, o estudo observacional de Schears et al., 2006 (Schears, 2006), apresentou especificamente uma taxa de flebite mecânica de 3,6\% com uso de adesivo comparado com $0,7 \%$ com o dispositivo de estabilização, o que seria responsável isoladamente por uma redução de aproximadamente $80 \%$ na taxa de flebite, apenas com a introdução de um dispositivo de estabilização integrado ao cateter periférico.

Adicionalmente, outras taxas de complicação importantes, incluindo oclusão, deslocamento, infiltração e extravasamento também apresentaram diferença significativa entre os grupos e merecem destaque.

Assim, a revisão apresenta, a despeito de algumas limitações dos estudos de base, uma tendência de que a estabilização dos cateteres periféricos, seja por dispositivos integrados ou avulsos, evite eventos adversos tais como extravasamento, deslocamento e flebite mecânica, entre outros. Ainda, esta revisão reforçou a necessidade da condução de novos estudos clínicos mais robustos para responder em definitivo à pergunta da revisão.

Na sequencia, foi conduzida análise econômica em saúde de forma a se determinar a custo-efetividade de dispositivos

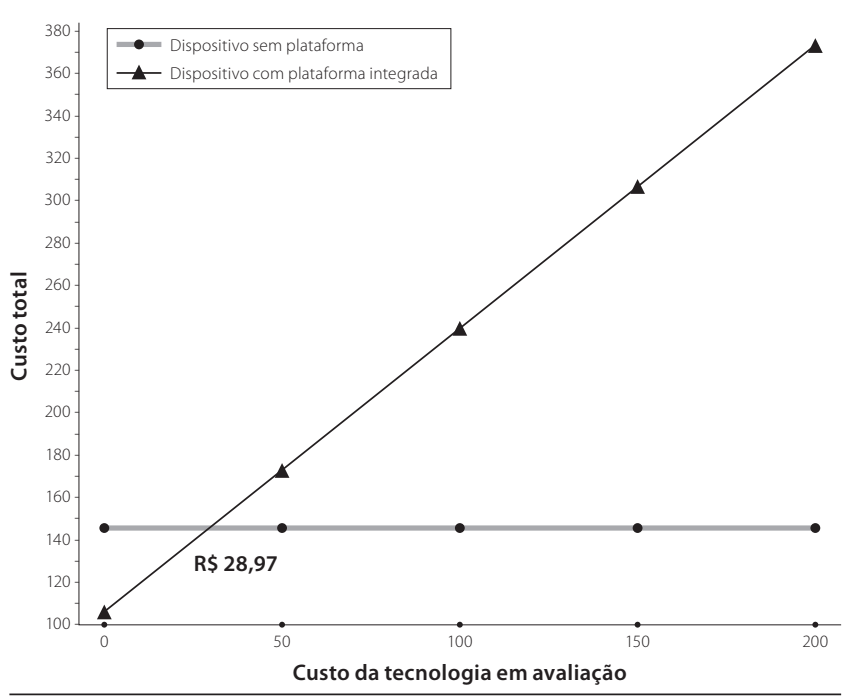

Figura 3. Análise de sensibilidade de preços para o cenário alternativo, desconsiderando-se os custos de aquisição dos comparadores.

com plataforma de estabilização integrada versus dispositivos sem plataforma. Pelo modelo analítico de decisão desenvolvido, fica evidente a economia de recursos financeiros que pode ser associada ao uso de dispositivos com plataforma integrada, redução de despesas esta associada a menor incidência de eventos adversos de segurança que podem onerar o custo total da terapia infusional do paciente.

\section{Conclusão}

Há clara tendência de que a estabilização dos cateteres periféricos, seja por dispositivos integrados ou avulsos, evite, por exemplo, extravasamento, deslocamento e flebite mecânica. Desta forma, o uso de cateteres periféricos com mecanismo ou plataforma de estabilização integrada pode contribuir para a redução de complicações, além de contribuir para diminuição do custo global relacionado, tomando como base as potenciais reduções de eventos adversos associados a mobilização inadvertida de cateteres periféricos e seus consequentes desdobramentos negativos.

\section{Limitações}

Os dados de eficácia clínica aplicados no modelo econômico discutido por este artigo são oriundos de revisões sistemáticas e metanálises recentes e de estudos randomizados que comparam diretamente as alternativas em destaque, publicados até a data de corte da etapa de revisão de literatura. Porém, não há como garantir que todos os estudos que se encaixaram nos critérios de inclusão tenham sido selecionados em função, sobretudo, de diferentes metodologias de indexação empregadas pelas bases de dados pesquisadas, do efeito gaveta (file drawer effect; estudos conduzidos, mas 
nunca publicados) e da literatura cinza (grey literature; informações produzidas e distribuídas em diversos níveis, em formato impresso ou eletrônico, não controladas, por publicações comerciais).

Com relação à validade externa do modelo, cabe ressaltar que todos os valores empregados para cálculo de materiais de consumo a partir dos Preços Fábrica publicados para o produto Introcan Safety ${ }^{\circledR}$, registrado e comercializado no Brasil pelos Laboratórios B.Braun S.A., o que pode determinar resultados diferentes, caso sejam considerados outros produtos e/ou sistemas concorrentes.

Por fim, não foi considerada a inclusão de dados referentes aos custos indiretos ou intangíveis, tampouco despesas com recursos humanos, em função destes vetores não serem apropriados à perspectiva da avaliação realizada.

\section{Referências bibliográficas}

Bausone-Gazda D, Cheryl A, Shelley-Ann WM. A randomized controlled trial to compare the complications of 2 peripheral intravenous catheter-stabilization systems. Journal of infusion nursing, v. 33, n. 6, p. 371-384, 2010.

BRASIL. Ministério da saúde. Secretaria de ciência- tecnologia e insumos estratégicos. Departamento de ciência e tecnologia. Diretrizes metodológicas: elaboração de pareceres técnico-científicos. Brasília: 2014.

BRASIL. Ministério da saúde. Secretaria de ciência-tecnologia e insumos estratégicos. Departamento de ciência e tecnologia. Diretrizes metodológicas: estudos de avaliação econômica de tecnologias em saúde. Brasília: Ministério da Saúde, 2009.

BRASÍNDICE. Guia Farmacêutico, Brasíndice 834. São Paulo: Andrei Publicações Médicas, 2015.

Campos L. S. Cómo reducir los riesgos de la punción venosa periférica con un nuevo catéter de sistema cerrado. ROL Enferm, v. 38, n. 4, p. 9-13, 2015.

CBHPM (Classificação Brasileira Hierarquizada de Procedimentos Médicos), 2014

CMED. Lista de preços de medicamentos: preço fábrica e preço máximo de venda ao governo. Agência Nacional de Vigilância Sanitária - Secretaria executiva.2015. Atualização: Junho/2015.

COFEN. Conselho federal de enfermagem. Tabela de honorários para prestação de serviçoes de enfermagem, 2012. Disponível em: <http:// al.corens.portalcofen.gov.br/wp-content/uploads/2013/04/Tabela-deHonor\%C3\%A1rios-para-Presta\%C3\%A7\%C3\%A3o-de-Servi\%C3\%A7osde-Enfermagem-2012.pdf.>.
Infusion Nurses Society. Infusion nursing standards of practice. J Infus Nurs., v. 29, n. suppl. 1, p. S1-S90, 2006.

IBGE. Instituto brasileiro de geografia e estatítica. PIB per capita. Disponível em: <http://cod.ibge.gov.br/4501p>.

Jacinto A, Avelar A, Wilson A. Phlebitis associated with peripheral intravenous catheters in children: study of predisposing factors. Escola Anna Nery - Revista de Enfermagem, v. 18, n. 2, p. 220-226, 2014.

Jadad A, Moore R, Carroll D, Jenkinson C, Reynolds D, Gavaghan D. Assessing the quality of reports of randomized clinical trials: is blinding necessary? Control Clin Trials., v. 17, n. 1, p. 1-12, 1996.

Laboratórios B.Braun S/A. Introcan Safety ${ }^{\oplus 3}$. 2013. p. 1-2.

Magerote N, Lima M, Silva J, Correia M, Secoli S. Associação entre flebite e retirada de cateteres intravenosos periféricos. Texto e Contexto Enfermagem, v. 20, n. 3, p. 286-292, 2011.

Marsh N, Webster J, Mihala G, Rickard C. Devices and dressings to secure peripheral venous catheters to prevent complications (Review). Cochrane Database of Systematic Reviews, n. 6, 2015.

MTE. NR 32 - Segurança e Saúde no Trabalho em Serviços de Saúde. Portaria GM 485/2005. 2005;(32):37.

OXFORD. Centre for evidence-based medicine. Oxford Centre for Evidencebased Medicine - Levels of Evidence (March 2009). Disponível em: $<$ http://www.cebm.net/oxford-centre-evidence-based-medicine-levelsevidence-march-2009/>.

Pasalioglu KB, Kaya H. Catheter indwell time and phlebitis development during peripheral intravenous catheter administration. Pakistan Journal of Medical Sciences, v. 30, n. 4, p. 725-730, 2014.

Schears GJ. Summary of product trials for 10, 164 patients: comparing an intravenous stabilizing device to tape. Journal of Infusion Nursing, v. 29, n. 4, p. 225-231, 2006

Shea B, Grimshaw J, Wells G, Boers M, Andersson N, Hamel C. et al. Development of AMSTAR: a measurement tool to assess the methodological quality of systematic reviews. BMC medical research methodology, v. 7, p. 10, 2007.

Shea B, Hamel C, Wells G, Bouter L, Kristjansson E, Grimshaw J. et al. AMSTAR is a reliable and valid measurement tool to assess the methodological quality of systematic reviews. Journal of clinical epidemiology, v. 62, n. 10, p. 1013-20, 2009

SIMPRO. Informações e soluções em saúde. Revista Simpro Hospitalar, 2015.

Souza A, Oliveira J, Dias D, Lazzari A. Prevalence of phlebitis in adult patients admitted to a university hospital. Revista da Rede de Enfermagem do Nordeste, v. 16, n. 1, p. 114-122, 2015.

Tamura N, Abe S, Hagimoto K, Kondo A, Matsuo A, Ozawa Y. et al. Unfavorable peripheral intravenous catheter replacements can be reduced using an integrated closed intravenous catheter system. The Journal of Vascular Access, v. 15, n. 4, p. 257-263, 2014. 